\title{
Function of IRAG for CGMP kinase signalling in smooth muscle and platelets Jens Schlossmann*
}

\author{
Address: Institut für Pharmakologie und Toxikologie der Technischen Universität München, Germany \\ Email: Jens Schlossmann* - Schlossmann@ipt.med.tu-muenchen.de \\ * Corresponding author
}

from 2nd International Conference of cGMP Generators, Effectors and Therapeutic Implications

Potsdam, Germany, 10-12 June, 2005

Published: 16 June 2005

BMC Pharmacology 2005, 5(SuppI I):SI3 doi:10.1I86/I47I-22I0-5-SI-SI3

Intracellular signalling by $\mathrm{NO} / \mathrm{cGMP} / \mathrm{cGMP}$-dependent protein kinase type I (cGKI) regulates various physiological processes including smooth muscle contractility and platelet aggregation. An important mediator of this signalling cascade is the inositol 1,4,5-trisphosphate receptor I $\left(\mathrm{IP}_{3} \mathrm{RI}\right)$ associated protein cGMP kinase substrate (IRAG). This protein forms a trimeric complex together with the cGMP kinase I $\beta$ (cGKI $\beta$ ) and the IP ${ }_{3}$ RI. Targeted deletion of exon 12 of IRAG coding for the N-terminal part of the coiled-coil domain disrupted in vivo the IRAG-IP ${ }_{3} \mathrm{RI}$ interaction. The resulting IRAG ${ }^{\Delta 12 / \Delta 12}$ mice showed an increased mortality and a severely reduced gastrointestinal motility. The relaxation of hormone-contracted aortic and longitudinal colonic smooth muscle by cGMP was abolished in IRAG ${ }^{\Delta 12 / \Delta 12}$ mice, whereas CAMP-mediated relaxation was not altered. In contrast to WT mice, norepinephrine-induced increases in $\left[\mathrm{Ca}^{2+}\right]_{\mathrm{i}}$ were not reduced by cGMP in aortic smooth muscle cells from IRAG ${ }^{\Delta 12 / \Delta 12}$ mice. These data suggest, that IRAG is involved in the cGMP-dependent decrease of $\left[\mathrm{Ca}^{2+}\right]_{\mathrm{i}}$ in vivo and is essential for cGMP-dependent relaxation of hormone-induced vascular and colonic muscle contraction. However, cGMP-mediated relaxation of small intestinal smooth muscles was only partially affected in IRAG ${ }^{\Delta 12 / \Delta 12}$ mice suggesting tissue specific selectivity of CGKI mechanisms.

In addition, IRAG is highly expressed in platelets. To study the effect of IRAG signalling in platelets, we analysed the aggregation of IRAG ${ }^{\Delta 12 / \Delta 12}$ platelets. Nitric oxide and the cGMP analogue 8-pCPT-cGMP did not inhibit the aggregation of IRAG ${ }^{\Delta 12 / \Delta 12}$ platelets in contrast to wild type platelets, whereas the shape change of platelets was not affected in both mutant and wild type platelets. Furthermore, tail bleeding was abbreviated in IRAG ${ }^{\Delta 12 / \Delta 12}$ mice suggesting a defect in the regulation of coagulation in vivo. Therefore, cGKI/IRAG/IP ${ }_{3}$ RI signalling might be crucial for the NO/cGMP-dependent inhibition of platelet aggregation. 